\title{
Novel strategies for the treatment of asthma
}

Marek Lommatzsch, Paul Stoll

Department of Pneumology and Critcal Care Medicine, University of Rostock, Germany

\begin{abstract}
Novel treatment strategies are currently emerging for patients with inadequately controlled asthma despite good adherence and trigger avoidance. These strategies serve primarily to reduce or completely avoid long-term oral corticosteroid therapy. A number of these options have already been implemented in practice or will soon be authorized for the treatment of asthma, while others still need to prove their clinical practicability, safety and efficacy.
\end{abstract}

\section{Asthma guidelines}

Current recommendations on asthma treatment are summarized in the international recommendations of the Global Initiative for Asthma (GINA), which were updated in 2015 (www.ginasthma. com). Revisions of the German asthma guidelines [1] from 2006 and the German national disease management guidelines (www.versorgungsleitlinien. de) will soon be published. It is controversial, however, whether the expensive and time-consuming development of national guidelines is still a sensible approach, given that these guidelines quickly become outdated due to the rapid progress of medical knowlegde. In addition, they might not be the preferred source of information for internet-oriented physicians.

\section{Asthma treatment: state of the art}

A distinction is made in asthma treatment between "controllers" (long-term treatment to control disease) and „relievers“ (as-needed treatment to control acute symptoms). The current GINA stepwise approach recommends treatment escalation until an optimal asthma control is reached. GINA step 1 recommends as-needed treatment only with a short-acting beta-agonist (SABA); alternatively, a low-dose inhaled corticosteroid (ICS) as a controller can already be considered at this stage (Fig. 1).
The present article provides an overview of the broad spectrum of novel inhaled, oral, systemic, and invasive treatment strategies for asthma.

Cite this as Lommatzsch M, Stoll P. Novel strategies for the treatment of asthma. Allergo J Int 2016;25: 11-7

DOI: 10.1007/s40629-015-0071-3

\section{Key words} step-up treatment scheme specific immunotherapy - inhaled therapy biologics thermoplasty

$\begin{array}{ll}\text { Abbreviations } \\ \text { ATS } & \text { American Thoracic Society } \\ \text { BMI } & \text { Body Mass Index } \\ \text { ERS } & \text { European Respiratory Society } \\ \text { FeNO } & \text { Fraction of exhaled nitric oxide } \\ \text { FEV1 } & \text { Forced expiratory volume in the first } \\ & \text { second } \\ \text { GINA } & \text { Global Initiative for Asthma } \\ \text { ICS } & \text { Inhaled corticosteroid } \\ \text { IgE } & \text { Immunoglobulin E } \\ \text { IL } & \text { Interleukin } \\ \text { LABA } & \text { Long-acting beta-agonist } \\ \text { LAMA } & \text { Long-acting muscarinic antagonist } \\ \text { MART } & \text { Maintenance and reliever therapy } \\ \text { OCS } & \text { Oral corticosteroid } \\ \text { OCT } & \text { Optical coherence tomography } \\ \text { SABA } & \text { Short-acting beta-agonist } \\ \text { SIT } & \text { Specific immunotherapy } \\ \text { Th } & \text { Thelper cells } \\ \text { TSLP } & \text { Thymic stromal lymphopoietin } \\ \end{array}$


Starting in GINA step 2 (e.g., frequent use of as-needed inhalations), ICS are first choice controllers, whereas montelukast or theophylline are (less effective) alternative controllers. At the next level of escalation, GINA 3, a combination of ICS with a second controller is recommended, preferably a long-acting beta antagonist (LABA; typically as an ICS/LABA fixed combination); montelukast or theophylline are alternative second controllers. According new GINA guidelines, ICS/LABA combinations - provided they contain formoterol - can also be used from GINA step 3 upwards as relievers (instead of SABA treatment) (MART concept: maintenance and reliever therapy). The principal feature of GINA step 4 is that it increases ICS/LABA combination therapy to the highest authorized dose. Furthermore, the long-acting inhaled anticholinergic (LAMA), tiotropium, which was approved in Germany for this indication in 2014 (only via the Respimat ${ }^{\circledR}$ inhaler), can be used as an add-on $[2,3]$. Since no asthma studies have been conducted to date for LAMA such as glycopyrronium, aclidinium, or umeclidinium, they have not yet been approved for asthma. Recent studies show that LAMA could represent an equal alternative to LABA as an ICS combination partner [4]. Thus, it is likely that there

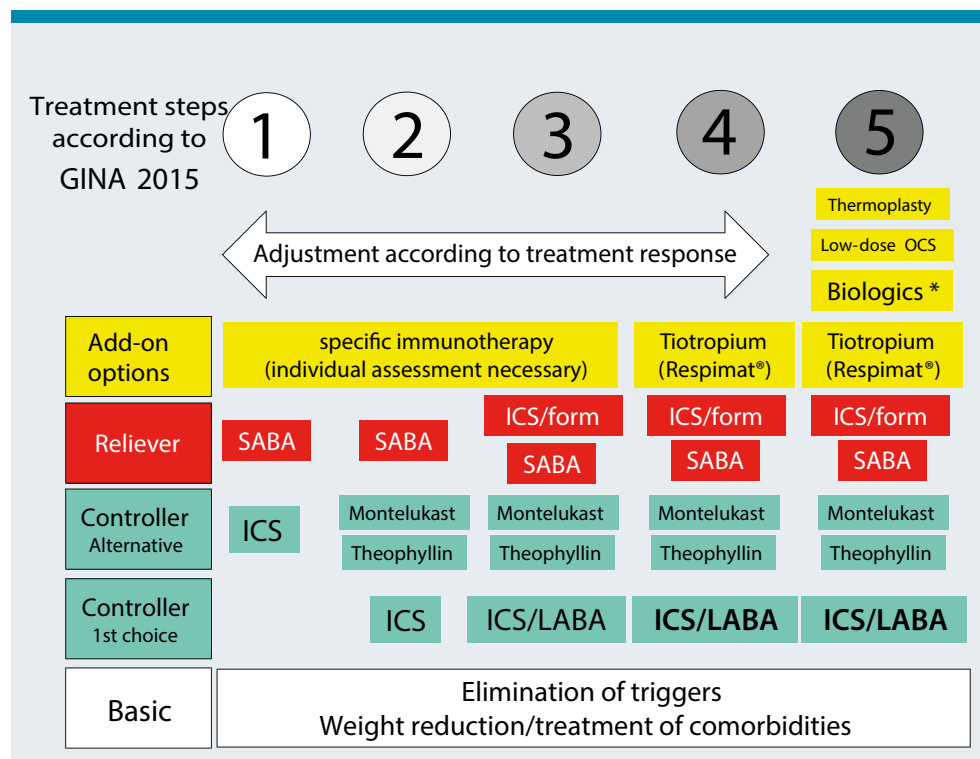

GINA, Global Initiative for Asthma; ICS, inhaled corticosteroid; ICS/Form, combination preparations comprising an ICS and formoterol; LABA, long-acting beta-agonist; OCS, oral corticosteroid; SABA, short-acting beta-agonist.

${ }^{*}$ Currently available biologics are anti-IgE (omalizumab) and anti-IL-5 (mepolizumab; the approval of the antibodies reslizumab und benralizumab is expected in the near future).

Fig. 1: Current asthma treatment according to GINA 2015 will be ICS/LAMA options in the future authorized for the use already in GINA treatment steps 2 and 3.

GINA step 5, in which several add-on treatment options come into play, is reached when high-dose ICS/LABA combination therapy, tiotropium therapy, and possibly concomitant oral therapy with montelukast and theophyllin fail to achieve adequate asthma control [5] (Fig. 1). However, before taking these additional options into consideration, it is important to ensure that patients and physicians get the following basics right [6]:

_ Is the patient receiving basic inhaled therapy that is tailored to the severity of disease?

- Is the patient handling the inhaler correctly and do they use the treatment regularly?

_Does the patient avoid triggers (e.g., cigarette smoking or allergen exposure) and contraindicated drugs (e.g., beta-blockers)?

- Is the patient receiving treatment for typical comorbidities (e.g. allergic rhinitis, gastroesophageal reflux, or obesity)?

_ Has the patient undergone training and/or rehabilitation?

Oral corticosteroid therapy (e.g., prednisolone) is often initiated when high-dose ICS/LABA therapy (and possibly additional controllers) fail to achieve asthma control. Inadequate asthma control, or the risk of losing asthma control under high-dose ICS/ LABA therapy and/or long-term prednisolone treatment, is classified as "severe asthma“ according to the current consensus definition of the American Thoracic Society (ATS) and European Respiratory Society (ERS) [5, 7]. The aim of all add-on options is to avoid (or at least reduce) long-term oral corticosteroid therapy without losing asthma control. Therefore, the current guideline postulates that addon options (in particular biologics such as anti-immunoglobulin E [IgE]) should be considered prior to prescribing a long-term therapy with oral corticosteroids.

Specific immunotherapy (SIT) is currently a rather theoretical option in severe asthma, since allergens directly associated with symptoms are rarely detected in this patient group and lung function is often below the forced expiratory volume in $1 \mathrm{sec}$ ond (FEV1) limit of $70 \%$ recommended for safety reasons. In addition, randomized studies on the efficacy of SIT in severe asthma are lacking. Thus, SIT usually represents an individual treatment option in GINA steps 1-3, in case of a clear relationship between clinical symptoms and allergen exposure (Fig. 1) $[8,9,10]$. Recent studies show that sublingual immuntherapy (SLIT) in patients with house dust mite allergy (and allergic rhinitis as a co-morbidity) can reduce the ICS dose and increase quality of life $[11,12]$. 


\section{Novel treatment approaches}

Most novel asthma treatment strategies have been developed as add-on options for patients who fail to achieve asthma control despite high-dose ICS/ LABA treatment. Some treatment approaches, however, are aimed at improving, or completely avoiding the need for standard asthma therapies (such as treatment with ICS or ICS/LABA combinations).

\section{Inhaled treatment options}

Switching conventional ICS therapy to inhalers with extra-fine formulations (average particle size: 1-3 $\mu \mathrm{m})$ can improve asthma control through better ICS deposition in the smaller airways: This option has already been established in clinical practice [13]. Another approach to optimize airway ICS deposition is the use of a computer-controlled inhalation system $\left(\mathrm{AKITA}^{\oplus}\right)$, which calculates the inhalation maneuver required for optimal particle deposition in a drug- and patient-specific manner. A randomized controlled trial in asthma patients treated with prednisolone recently showed that high-dose budesonide (1 mg twice daily) administered with the AKITA ${ }^{\oplus}$ inhalation system significantly reduced the need for prednisolone treatment while improving lung function and quality of life [14]. Novel inhaled anti-inflammatory strategies are under investigation, both as add-on options and as alternatives to ICS therapy. The inhalation of DNAzyme (also via AKITA ${ }^{\oplus}$ to inhibit GATA-3, an essential transcription factor for Type 2 immune responses, currently represents the most innovative approach [15]. It poses considerable challenges not only for the company developing the treatment, but also for the regulatory authorities, since both the target (GATA-3) and the mode of therapy (inhalation of DNAzyme) are completely new. Because of positive proof-ofconcept data [13] phase-IIb and phase-III studies are expected in the near future.

\section{Oral treatment options}

Immunomodulation with macrolides is currently not recommended in severe asthma due to its potential side effects (ototoxicity, QT interval prolongation, development of macrolide resistance) and the paucity of clinical data [7]. However, the recent AZISAST trial showed that the macrolide azithromycin (3 x $250 \mathrm{mg} /$ week; initial dose, $250 \mathrm{mg} /$ day for 5 days) reduces the risk of exacerbation in non-eosinophilic severe asthma [16]. The opposite effect was observed in patients with eosinophilic asthma (increased exacerbation risk under therapy) [16]. Thus, azithromycin treatment should only be considered in the case of frequent exacerbations and a low blood eosinophil count $(<200 / \mu$ l without systemic corticosteroids). The phosphodiesterase 4 inhibitor roflumilast showed clinical efficacy in a small study in patients with mild to moderate asthma [17]. However, there are no studies available for severe asthma. The efficacy of this treatment in severe asthma is, therefore, still open. Prostaglandin D2 receptor (CRTH2) antagonists (such as setipiprant [18] or fevipiprant [19]) are currently being investigated as novel oral anti-inflammatory treatment strategies in asthma patients.

\section{Biologics}

Add-on treatment with omalizumab administered subcutaneously has been authorized in Germany since 2005 in patients with severe allergic asthma (and a total IgE of 30-1500 kU/l serum; lower cutoffs apply above a body weight of $50 \mathrm{~kg}$ ). The efficacy of this treatment was demonstrated not only in randomized controlled trials, but also in real-life studies [20, 21]. Recent studies suggest, however, that omalizumab is also effective in intrinsic asthma (patients in whom an allergic sensitization cannot be found) [22]. There are two possible explanations for this. On the one hand, intrinsic asthma patients could be allergic to unknown allergens. On the other hand, the reduction of exacerbations following anti-IgE treatment could be due to improved antiviral immune responses (mediated by a reduced expression of high-affinity IgE receptors on plasmacytoid dendritic cells), thus making the effect independent of a specific allergy [22]. It appears that the anti-IgE effect in asthma is not only based on a reduction in IgE-mediated mast cell degranulation, but also a broad and sustained immunomodulation [23] (Fig. 2). Clinical studies with ligelizumab, a monoclonal antibody with significantly higher IgE affinity compared with omalizumab [24], in patients with asthma have recently been stopped, for yet unknown reasons.

Initial studies with antibodies inhibiting interleukin-5 (IL-5), a cytokine essential for the maturation, survival, and recruitment of eosinophilic granulocytes, were negative since no attention was paid to asthma severity and eosinophil counts [25]. However, after focussing on patients with severe asthma and elevated eosinophil counts in sputum, it became clear that this approach is a valuable add-on in patients with severe eosinophilic asthma $[26,27]$. The DREAM study (2012) was the first to open up the way to identifying patients that benefit from this treatment using differential blood counts (rather than less practicable and poorly available sputum diagnostics) [28]. The MENSA study (2014) was the first to demonstrate that the anti-IL-5 antibody mepolizumab can also be administered subcutaneously and that this therapy not only reduced the exacerbation rate, but also improved lung function [29]. Finally, the SIRIUS study (2014) showed that, in patients with prednisolone tretament, mepolizumab 


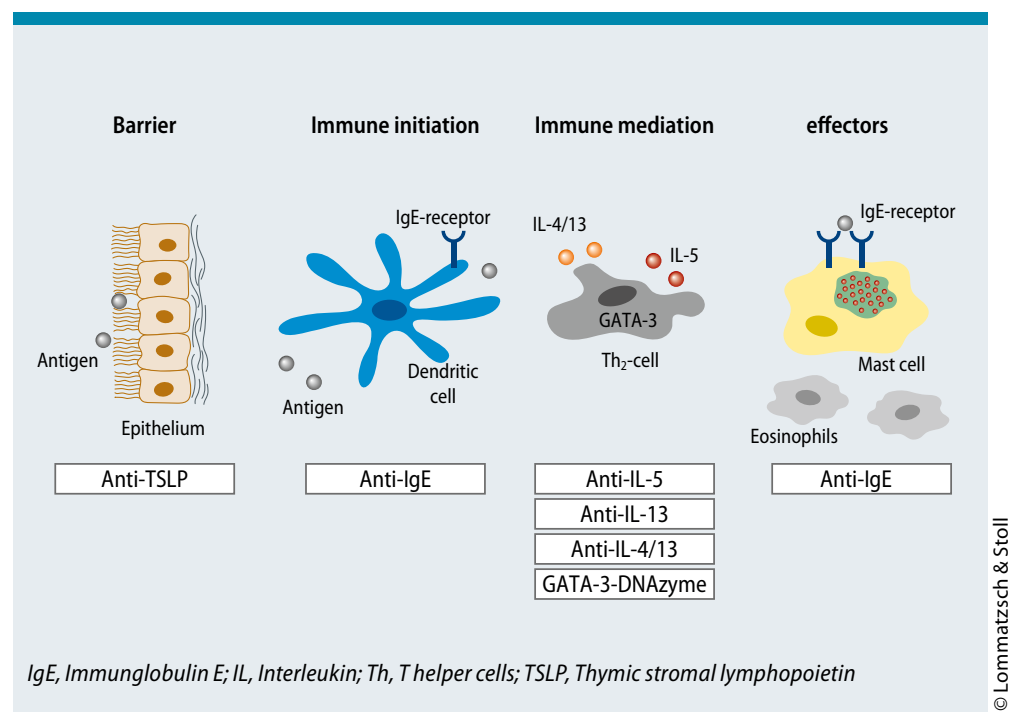

Fig. 2: Biologics for the treatment of asthma. There is a broad spectrum of targets, ranging from influencing epithelial mediators to modulating the inflammatory pathway. A number of biological drugs, such as the antiimmunoglobulin $\mathrm{E}$ (IgE) antibody omalizumab, have several sites of action. resulted in significant reduction in the prednisolone dose while at the same time improving asthma control [30]. Thus, the concept of an anti-IL-5 therapy has become real and practicable over the last 15 years (Fig. 2). Parallel to mepolizumab, two other antibodies, the anti-IL-5 antibody reslizumab [31] and the anti-IL-5 receptor antibody benralizumab [32], are currently under investigation in clinical trials. At present, these antibodies differ primarily in terms of the blood eosinophil cut-off values chosen in the studies for patient selection: $>150-300 / \mu \mathrm{l}$ for mepolizumab, $>300 / \mu$ l for benralizumab, and $>400 / \mu \mathrm{l}$ for reslizumab. The optimal blood eosinophil cut-off value is currently unclear. The german summary of product characteristics for mepolizumab avoids mentioning one defined cut-off: instead, 3 studies (DREAM, MENSA, SIRIUS) are reported, which used different inclusion criteria and blood eosinophil cut-offs. Furthermore, it is currently also unclear whether inhibition of the IL- 5 receptor (benralizumab) is clinically more effective than is inhibition of IL-5 (mepolizumab and reslizumab). However, there is no doubt that this new therapeutic concept will change clinical practice [33]. Mepolizumab has already been approved for the treatment of severe eosinophilic asthma in germany, the approval of reslizumab and benralizumab is expected in the near future.

Inhibition of the Th2 cytokines IL- 4 and IL-13 has long be the subject of asthma research. Inhib- iting IL-4 alone has not yielded convincing clinical effects as yet [34]. The IL-13 antagonists, lebrikizumab and tralokinumab, improved lung function in patients with severe asthma, particularly in the subgroup of patients with elevated periostin levels $[35,36]$ (periostin is produced in the respiratory epithelium in an IL-13-dependent manner [37]). However, clinical studies with anti-IL-13 antibodies conducted to date have failed to show a significant improvement in quality of life or reduction in exacerbation rates $[35,36]$. Thus, the future significance of IL-13 antibody therapy remains unclear. Great hopes are pinned on dupilumab, an antibody that inhibits the effect of both IL-4 and IL-13 [38]. A proof-of-concept study showed that subcutaneous administration of this antibody can partially substitute ICS/LABA therapy in patients with severe eosinophilic asthma [38]. A recent study on the efficacy of dupilumab as an ICS/LABA add-on therapy in patients with severe asthma (inclusion criteria: FEV1 40\%-80\% predicted; at least one exacerbation in the previous 12 months; no systemic therapy with corticosteroids) showed that dupilumab improves lung function and reduces exacerbation rates. Although this effect was most pronounced in patients with high blood eosinophil levels (> 300 eosinophils $/ \mu 1$ blood), it was nevertheless also significant in patients with low blood eosinophil counts $(<300$ eosinophils / $\mu$ l blood) [39]. Of note, dupilumab also reduced exhaled nitric oxide (FeNO) levels [39], an effect not observed under anti-IL-5 therapy [26].

Anti-TSLP antibody treatment is a completely new concept for the tretment of asthma. Thymic stromal lymphopoietin (TSLP) is an epithelial mediator that plays an important role in the initiation of inflammatory processes of the airways (e.g., by modulating dendritic cell function). Intravenous therapy with the anti-TSLP antibody AMG 157 reduced early and late allergic responses following allergen challenge in patients with allergic asthma [40]. Therefore, further clinical studies in patients with asthma, as well as patients with other allergic diseases, are planned [41].

\section{Invasive treatment options}

Asthma is often accompanied by neuromuscular hyperresponsiveness in the airways [42]. Since this is considered the result of the chronic inflammatory process, treating the underlying inflammation represents the primary target in asthma therapy (see treatment strategies discussed above). However, there is also the concept to directly treat the affected neuromuscular apparatus of the airways. The aim of bronchial thermoplasty is to irreversibly weaken the neuromuscular apparatus of the airways by means of endobronchial radiofrequency ablation 
using a special catheter. The placebo-controlled AIR2 trial showed that bronchial thermoplasty achieves a sustained reduction in exacerbations and hospitalizations in patients with severe asthma [43, 44]. Two histological studies postulated that this clinical effect is based on a direct destruction of smooth muscles $[45,46]$. However, smooth muscle was also reduced in untreated pulmonary lobes [45] and did not correlate with the intensity of bronchial thermoplasty treatment [46]. Therefore, the mechanisms underlying the clinical effects of bronchial thermoplasty still remain enigmatic. It is conceivable that bronchial thermoplasty affects the innervating nerves of the airways [47], however, this hypothesis has not yet been explored. To what extent optical coherence tomography (OCT), a minimally invasive procedure to visualize the airway wall, will shed light on the local effects of thermoplasty remains to be seen [48]. There is currently no consensus in Germany on patient selection for thermoplasty. In the opinion of the authors, this treatment should be reserved for those patients in whom all the aforementioned drug and conservative treatment strategies have been carefully explored. Due to the unclear mode of action and unknown long-term effects of bronchial thermoplasty, international guidelines recommend that this treatment be carried out only in the context of clinical studies or independent systematic registries [7].

Obesity is a common cause of treatment-refractory asthma [49]. Weight reduction by dieting improves asthma control and lung function [50]. However, conservative weight reduction measures often fail. Therefore, bariatric surgery to improve asthma control is currently under discussion. A recent study showed that bariatric surgery in patients with a body mass index (BMI) of $>35 \mathrm{~kg} / \mathrm{m}^{2}$ significantly improved lung function, asthma control, and bronchial hyperresponsiveness [51]. Further studies are needed to assess the real-world value of this surgical intervention in obese asthma patients.

\section{Summary}

The standards and concepts in asthma treatment are changing rapidly. New inhaled, oral, systemic and invasive treatment options, which are currently explored in clinical studies or have already been approved by the authorities, will change clinical practice.

\section{Prof. Dr. Marek Lommatzsch}

Department of Pneumology and Interdisciplinary Internal Intensive Care Unit

Medical Clinic I, Center for Internal Medicine

Rostock University Hospital

Ernst-Heydemann-Straße 6, 18057 Rostock, Germany

E-Mail: marek.lommatzsch@med.uni-rostock.de

\section{Conflicts of interest}

Prof. Lommatzsch has received honoraria for lectures, honoraria for advisory activities, travel costs and participation fee from ALK Abelló, Allergopharma, Astra Zeneca, Bencard, Berlin-Chemie, Boehringer-Ingelheim, Boston Scientific, Chiesi, GSK, Janssen-Cilag, MSD, Mundipharma, Novartis, Nycomed/Takeda, TEVA and UCB. Furthermore he has received honoraria for clinical studies from Astra Zeneca and research support from Firma GSK. Dr. Stoll has received honoraria for advisory activities and lectures from Astra Zeneca, Bayer, Berlin-Chemie, BoehringerIngelheim and Novartis.

\section{Cite this as}

Lommatzsch M, Stoll P. Novel strategies for the treatment of asthma. Allergo J Int 2016;25:11-7

DOI: 10.1007/s40629-015-0071-3

\section{References}

1. Buhl R, Berdel D, Criee CP et al. Guidelines for diagnosis and treatment of asthma patients. Pneumologie 2006;60:139-77

2. Kerstjens HA, Disse B, Schroder-Babo W et al. Tiotropium improves lung function in patients with severe uncontrolled asthma: a randomized controlled trial. J Allergy Clin Immunol 2011;128:308-14

3. Kerstjens HA, Engel M, Dahl R et al. Tiotropium in asthma poorly controlled with standard combination therapy. $\mathrm{N}$ Engl J Med 2012;367:1198-207

4. Kerstjens HA, Casale TB, Bleecker ER et al. Tiotropium or salmeterol as add-on therapy to inhaled corticosteroids for patients with moderate symptomatic asthma: two replicate, double-blind, placebo-controlled, parallel-group, active-comparator, randomised trials. Lancet Respir Med 2015;3:367-76

5. Lommatzsch M, Virchow CJ. Severe asthma: definition, diagnosis and treatment. Dtsch Arztebl Int 2014;111:84755

6. Bush A, Pavord ID. Omalizumab: NICE to USE you, to LOSE you NICE. Thorax 2013;68:7-8

7. Chung KF, Wenzel SE, Brozek JL et al. International ERS/ ATS guidelines on definition, evaluation and treatment of severe asthma. Eur Respir J 2014;43:343-73

8. Abramson MJ, Puy RM, Weiner JM. Allergen immunotherapy for asthma. Cochrane Database Syst Rev 2000:CD001186

9. Jutel M, Agache I, Bonini S et al. International consensus on allergy immunotherapy. J Allergy Clin Immunol 2015;136:556-68

10. Pfaar O, Bachert C, Bufe A, Buhl R, Ebner C, Eng P et al. Guideline on allergen-specific immunotherapy in IgE mediated allergic diseases - S2k Guideline of the German Society for Aller gology and Clinical Immunology (DGA$\mathrm{KI})$, the Society for Pediatric Allergy and Environmental Medicine (GPA), the Medical Association of German Allergologists (AeDA), the Austrian Society for Allergy and Immunology (ÖGAI), the Swiss Society for Allergy and Immunology (SGAI), the German Society of Dermatology (DDG), the German Society of Oto-Rhino-Laryngology, 
Head and Neck Surgery (DGHNO-KHC), the German Society of Pediatrics and Adolescent Medicine (DGKJ), the Society for Pediatric Pneumology (GPP), the German Respiratory Society (DGP), the German Association of ENT Surgeons (BV-HNO), the Professional Federation of Paediatricians and Youth Doctors (BVKJ), the Federal Association of Pulmonologists (BDP) and the German Dermatologists Association (BVDD). Allergo J Int 2014;23:282-319

11. Mosbech H, Deckelmann R, de Blay F, Pastorello EA, Trebas-Pietras E, Andres LP et al. Standardized quality (SQ) house dust mite sublingual immunotherapy tablet (ALK) reduces inhaled corticosteroid use while maintaining asthma control: a randomized, double-blind, placebo-controlled trial. J Allergy Clin Immunol 2014;134:56875

12. de Blay F, Kuna P, Prieto L, Ginko T, Seitzberg D, Riis B et al. SQ HDM SLIT-tablet (ALK) in treatment of asthma-post hoc results from a randomised trial. Respir Med 2014; 108:1430-7

13. Berge $\mathrm{M}$ van den, Hacken $\mathrm{NH}$ ten, Wiel $\mathrm{E}$ van der, Postma DS. Treatment of the bronchial tree from beginning to end: targeting small airway inflammation in asthma. Allergy 2013;68:16-26

14. Vogelmeier $C$, Kardos $P$, Hofmann T et al. Nebulised budesonide using a novel device in patients with oral steroid-dependent asthma. Eur Respir J 2015;45:1273-82

15. Krug N, Hohlfeld JM, Kirsten AM et al. Allergen-induced asthmatic responses modified by a GATA3-specific DNAzyme. N Engl J Med 2015;372:1987-95

16. Brusselle GG, Vanderstichele C, Jordens P et al. Azithromycin for prevention of exacerbations in severe asthma (AZISAST): a multicentre randomised double-blind placebo-controlled trial. Thorax 2013;68:322-9

17. Bousquet J, Aubier M, Sastre J et al. Comparison of roflumilast, an oral anti-inflammatory, with beclomethasone dipropionate in the treatment of persistent asthma. Allergy $2006 ; 61: 72-8$

18. Diamant Z, Sidharta PN, Singh D et al. Setipiprant, a selective CRTH2 antagonist, reduces allergen-induced airway responses in allergic asthmatics. Clin Exp Allergy 2014;44:1044-52

19. Luu VT, Goujon JY, Meisterhans C, Frommherz M, Bauer C. Synthesis of a high specific activity methyl sulfone tritium isotopologue of fevipiprant (NVP-QAW039). J Labelled Comp Radiopharm 2015;58:188-95

20. Humbert M, Beasley R, Ayres J et al. Benefits of omalizumab as add-on therapy in patients with severe persistent asthma who are inadequately controlled despite best available therapy (GINA 2002 step 4 treatment): INNOVATE. Allergy 2005;60:309-16

21. Braunstahl GJ, Chen CW, Maykut R, Georgiou P, Peachey $G$, Bruce J. The eXpeRience registry: the 'real-world' effectiveness of omalizumab in allergic asthma. Respir Med 2013;107:1141-51

22. Lommatzsch M, Korn S, Buhl R, Virchow JC. Against all odds: anti-IgE for intrinsic asthma? Thorax 2014;69:94-6

23. Humbert M, Busse W, Hanania NA et al. Omalizumab in asthma: an update on recent developments. J Allergy Clin Immunol Pract 2014;2:525-36 e1

24. Arm JP, Bottoli I, Skerjanec A et al. Pharmacokinetics, pharmacodynamics and safety of QGE031 (Ligelizumab), a novel high-affinity anti-IgE antibody, in atopic subjects. Clin Exp Allergy 2014;44:1371-85

25. Leckie MJ, Brinke A ten, Khan J et al. Effects of an interleukin-5 blocking monoclonal antibody on eosinophils, airway hyper-responsiveness, and the late asthmatic response. Lancet 2000;356:2144-8

26. Haldar $\mathrm{P}$, Brightling $\mathrm{CE}$, Hargadon $\mathrm{B}$ et al. Mepolizumab and exacerbations of refractory eosinophilic asthma. $\mathrm{N}$ Engl J Med 2009;360:973-84

27. Pavord ID, Haldar P, Bradding P, Wardlaw AJ. Mepolizumab in refractory eosinophilic asthma. Thorax 2010;65:370
28. Pavord ID, Korn S, Howarth P et al. Mepolizumab for severe eosinophilic asthma (DREAM): a multicentre, double-blind, placebo-controlled trial. Lancet 2012;380:6519

29. Ortega HG, Liu MC, Pavord ID et al. Mepolizumab treatment in patients with severe eosinophilic asthma. $\mathrm{N} \mathrm{Engl}$ J Med 2014;371:1198-207

30. Bel EH, Wenzel SE, Thompson PJ et al. Oral glucocorticoid-sparing effect of mepolizumab in eosinophilic asthma. N Engl J Med 2014;371:1189-97

31. Castro M, Zangrilli J, Wechsler ME et al. Reslizumab for inadequately controlled asthma with elevated blood eosinophil counts: results from two multicentre, parallel, double-blind, randomised, placebo-controlled, phase 3 trials. Lancet Respir Med 2015;3:355-66

32. Castro $\mathrm{M}$, Wenzel SE, Bleecker ER et al. Benralizumab, an anti-interleukin 5 receptor alpha monoclonal antibody, versus placebo for uncontrolled eosinophilic asthma: a phase $2 \mathrm{~b}$ randomised dose-ranging study. Lancet Respir Med 2014;2:879-90

33. Legrand F, Klion AD. Biologic therapies targeting eosinophils: current status and future prospects. J Allergy Clin Immunol Pract 2015;3:167-74

34. Hambly N, Nair P. Monoclonal antibodies for the treatment of refractory asthma. Curr Opin Pulm Med 2014;20:87-94

35. Corren J, Lemanske RF, Hanania NA et al. Lebrikizumab treatment in adults with asthma. N Engl J Med 2011;365:1088-98

36. Brightling CE, Chanez P, Leigh R et al. Efficacy and safety of tralokinumab in patients with severe uncontrolled asthma: a randomised, double-blind, placebo-controlled, phase $2 \mathrm{~b}$ trial. Lancet Respir Med 2015;3:692-701

37. Li W, Gao P, Zhi Y et al. Periostin: its role in asthma and its potential as a diagnostic or therapeutic target. Respir Res 2015;16:57

38. Wenzel S, Ford L, Pearlman D et al. Dupilumab in persistent asthma with elevated eosinophil levels. N Engl J Med 2013;368:2455-66

39. Wenzel $S$, Wang $L$, Pirozzi $G$ et al. Dupilumab improves lung function and reduces severe exacerbations in uncontrolled asthmatics with baseline eosinophil levels above and below 300 Cells/ $\mu \mathrm{L}$. Am J Respir Crit Care Med 2015;191:A6362

40. Gauvreau GM, O'Byrne PM, Boulet LP et al. Effects of an anti-TSLP antibody on allergen-induced asthmatic responses. N Engl J Med 2014;370:2102-10

41. Cianferoni A, Spergel J. The importance of TSLP in allergic disease and its role as a potential therapeutic target. Expert Rev Clin Immunol 2014;10:1463-74

42. Lommatzsch M. Airway hyperresponsiveness: new insights into the pathogenesis. Semin Respir Crit Care Med 2012;33:579-87

43. Castro M, Rubin AS, Laviolette $M$ et al. Effectiveness and safety of bronchial thermoplasty in the treatment of severe asthma: a multicenter, randomized, double-blind, sham-controlled clinical trial. Am J Respir Crit Care Med 2010;181:116-24

44. Wechsler ME, Laviolette M, Rubin AS et al. Bronchial thermoplasty: Long-term safety and effectiveness in patients with severe persistent asthma. J Allergy Clin Immunol 2013;132:1295-302

45. Pretolani M, Dombret MC, Thabut $\mathrm{G}$ et al. Reduction of airway smooth muscle mass by bronchial thermoplasty in patients with severe asthma. Am J Respir Crit Care Med 2014;190:1452-4

46. Chakir J, Haj-Salem I, Gras D et al. Effects of bronchial thermoplasty on airway smooth muscle and collagen deposition in asthma. Ann Am Thorac Soc 2015 (online first)

47. Lommatzsch M, Virchow JC. The neural underpinnings of asthma. J Allergy Clin Immunol 2007;119:254-5; author reply 5 
48. Kirby M, Ohtani K, Lopez Lisbona RM et al. Bronchial thermoplasty in asthma: 2-year follow-up using optical coherence tomography. Eur Respir J 2015;46:85962

49. Mosen DM, Schatz M, Magid DJ, Camargo CA Jr. The relationship between obesity and asthma severity and control in adults. J Allergy Clin Immunol 2008;122:50711 e6
50. Pakhale S, Baron J, Dent R, Vandemheen K, Aaron SD. Effects of weight loss on airway responsiveness in obese adults with asthma: does weight loss lead to reversibility of asthma? Chest 2015;147:1582-90

51. Huisstede A van, Rudolphus A, Castro Cabezas M et al. Effect of bariatric surgery on asthma control, lung function and bronchial and systemic inflammation in morbidly obese subjects with asthma. Thorax 2015;70:659-67 